\title{
Blended antilock braking system control method for all-wheel drive electric sport utility vehicle
}

\author{
Andrei Aksjonov $\cdot$ Valery Vodovozov $\cdot$ Klaus Augsburg $\cdot$ Eduard Petlenkov
}

\begin{abstract}
At least two different actuators work in cooperation in regenerative braking for electric and hybrid vehicles. Torque blending is an important area, which is responsible for better manoeuvrability, reduced braking distance, improved riding comfort, etc. In this paper, a control method for electric vehicle blended antilock braking system based on fuzzy logic is promoted. The principle prioritizes usage of electric motor actuators to maximize recuperation energy during deceleration process. Moreover, for supreme efficiency it considers battery's state of charge for switching between electric motor and conventional electrohydraulic brakes. To demonstrate the functionality of the controller under changing dynamic conditions a hardware-in-the-loop simulation with real electrohydraulic brakes test bed is utilized. In particular, the experiment is designed to exceed the state of charge threshold during braking operation, what leads to immediate switch between regenerative and friction brake modes.
\end{abstract}

\section{Introduction}

One of the advantageous features of the electric vehicles (EVs) is their ability to recuperated energy during a

\section{A. Aksjonov}

ŠKODA Auto a.s.

Mladá Boleslav, Czech Republic

E-mail: andrei.aksjonov@skoda-auto.cz

\section{K. Augsburg}

Technische Universität Ilmenau

Ilmenau, Germany

E-mail: klaus.augsburg@tu-ilmenau.de

V. Vodovozov $\cdot$ E. Petlenkov

Tallinn University of Technology

Tallinn, Estonia

E-mail: valery.vodovozov@taltech.ee,eduard.petlenkov@taltech.ee deceleration process. In EVs, friction braking (FB) cooperates with regenerative braking $(\mathrm{RB})$, what opens a need to efficient torque control between two separate actuators (i.e. torque blending), which are characterized by different dynamics. In some cases, RB is simply not enough to achieve requested braking torque, therefore, the FB system is activated in parallel or in series. In other case, the battery conditions (e.g. temperature, battery's state of charge (SOC), etc.) must be considered. For instance, when the battery is fully charged, the recuperation is no longer useful and even dangerous [1].

The SOC is a ratio of the remaining battery capacity to the full charged one. It is one of the most important parameters in EVs. Its feature is used not only in battery management to estimate potential driving range before the next recharge, but also in vehicle traction (e.g. hybrid EV) and braking (e.g. blended braking system) control strategies [1]. For example, to avoid electric battery overcharge, and consequent damage, the regeneration by electric motors is usually limited to a specific upper bound, 80-90\% [2]. Therefore, the SOC must be always involved in a blended antilock braking system (ABS).

Nowadays, the fuzzy logic controllers (FLCs) are widely used in automotive engineering to solve various problems [3]. For instance, in [4], an effectiveness and strong robustness of a fuzzy sliding mode control over conventional proportional-integral-derivative (PID) and Mamdani's type FLC in energy recuperation for EV in simulation environment was demonstrated. Complexity of vehicle dynamics in deceleration process, especially during emergency braking, was not integrated in the study.

An FLC-based RB strategy integrated with series RB was developed in [5]. The FLC received driver's force command, vehicle speed, battery's SOC and temperature to determine distribution between $\mathrm{FB}$ and $\mathrm{RB}$ to improve 
energy recuperation efficiency. In [6], the FLC involved SOC and a ratio between brake torque and biggest brake torque to determine factual $\mathrm{FB}$ and RB brake torques. An $\mathrm{RB}$ control strategy applying FLC was presented in [7]. The simulation results demonstrated that the developed method is able to recover energy and distribute power flow to maintain SOC around target value. A PID in combination with FLC ensured efficient RB strategy of the EV [8]. The SOC was taken as an input of the FLC. Despite impressive results, all these works only focused on the base brake case. The ABS function was not considered.

In [9], the authors applied genetic algorithm in EV stability control logic using RB of the rear wheels motor and FB of electrohydraulic brake (EHB). The simulation results showed that the optimal recuperation strategy is able to provide an increase of recuperation energy. However, neither SOC in torque allocation nor ABS performance were under investigation. Brake force distribution strategy for EVs based on estimation of tire-road friction coefficient was provided in [10]. The road condition estimation was also based on fuzzy theory. An efficient torque blending was demonstrated in [11]. The experiment was conducted on a real vehicle braking on low-friction road surface. In [12], the FLC was used to adjust braking torque between RB and FB. However, in these works torque blending or force distribution did not consider SOC of a battery.

Scholars in [13] integrated sliding mode controller with FLC for an ABS control to maintain optimal wheel slip ratio deceleration. The SOC was reckoned in torque blending in this instant. Nevertheless, for the ABS control method the reference slip was fixed, thus, changing optimal slip for various road conditions was not involved. Advanced control allocation with energy recuperation for EV was introduced in [14]. The authors also involved battery's SOC. Both works did not study the situation, when SOC exceeds its bound during braking manoeuvre.

Earlier [15], the EV torque blending with recuperation capabilities with SOC taken into account was proposed by the authors. It was integrated with three types of controllers, namely PID, tabular, and FLC. In this paper, the attention is once again focused on SOC's influence on EV's blended ABS. To this aim, the intelligent FLC control method previously developed by the authors [16] is applied in hardware-in-the-loop (HIL) simulation with real EHB system. The HIL testbed accompanied with a hardware delay is exploited to represent actual EHB dynamics, making the simulation experiment more valued for real life application. The deceleration test is designed in a way that SOC reaches its maximum threshold in the middle of the braking process. Consequently, blended braking system rapidly switches from $\mathrm{RB}$ to $\mathrm{FB}$.

In $\mathrm{RB}$, the recovered energy is not stored directly in the battery, but in the ultracapacitor. From the latter, the recuperated energy is transmitted slowly to the battery or is used for vehicle acceleration. Thus, the SOC shall also consider capacity of an ultracapacitor. Furthermore, the electronic power converters play an essential role in energy recuperation in EVs as they are an intermediate connection between energy sources and motors. In this paper, it is assumed that the energy is transferred directly to the battery, thus, the SOC may surpass its maximum limit during the EV deceleration. However, the power electronics loses are neglected in the powertrain model. Nevertheless, possible consideration of the SOC of ultracapacitor in torque blending was also proposed by the authors in [15].

This paper is organized as follows. The next section stresses the HIL simulation environment together with vehicle modeling. Section III describes the blended ABS control method. In Section IV, the HIL simulation results are delivered. The paper is briefly concluded in Section V.

\section{Vehicle model and experimental setup}

\subsection{Single wheel model}

A simplified schematic single wheel brake diagram is drawn in Fig. 1. The rolling resistance and lateral dynamics are neglected, because only the straight braking manoeuvre is studied in this work. The torque balance about a wheel axis is expressed as:

$J_{w} \dot{\omega}_{w}=T_{d}-r_{w} \cdot F_{x}-T_{b}$,

where $J_{W}-$ moment of inertia of wheel; $\omega_{W}-$ angular velocity of wheel; $T_{b}$ - braking torque; $T_{d}$ - driving torque; $r_{W}$ - radius of deformed tire; $F_{x}$ - longitudinal force of tire.

A distinctive feature of the EV: its braking torque $T_{b}$ is a summation of the RB $T_{R B}$ and FB $T_{F B}$ braking torques [16]:

$$
T_{b}=T_{F B}+T_{R B} \text {. }
$$

In practice, $T_{R B}$ and $T_{F B}$ are not measured by the sensors directly. They change proportionally to phase current of a switched reluctance motor (SRM) and line pressure of an EHB, accordingly. Those states are measured by available on-board sensors in modern vehicles. In this paper, the variables are represented as torques directly.

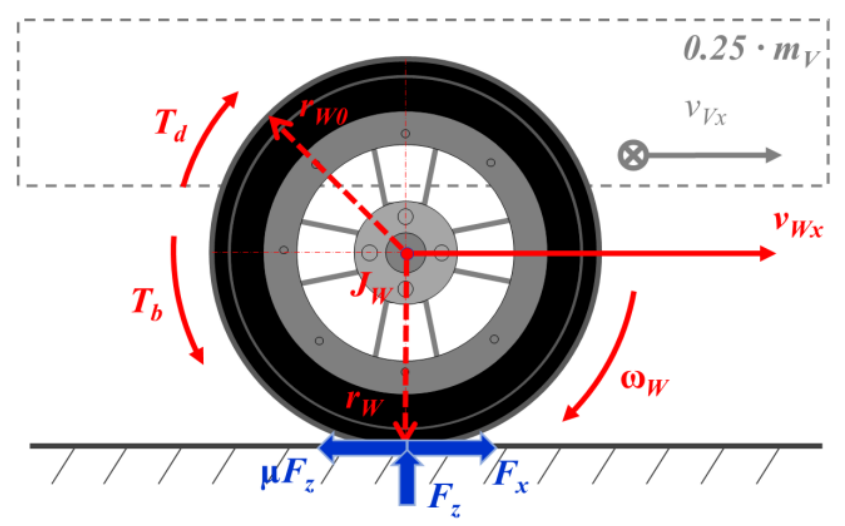

Fig. 1 A schematic drawing of a braked wheel for a single-wheel model. 


\subsection{State estimation}

An essential characteristic of an ABS is tire-road friction coefficient $\mu$. Straight direction braking manoeuvre neglects lateral dynamics, hence, $\mu$ is calculated as a ratio of longitudinal $F_{x}$ and normal $F_{z}$ forces:

$$
\mu=\frac{F_{\chi}}{F_{z}} \text {. }
$$

The proposed control method uses $\mu$ to understand the road surface under the tires of the EV. In this regard, $\mu$ is assumed to be proportional to the EV body deceleration rate [16]:

$$
\mu^{*}=\frac{F_{x}}{F_{z}}=\frac{m_{v} \cdot a_{V x}}{m_{v} \cdot g}=\frac{a_{V x}}{g},
$$

where $m_{v}$ - mass of vehicle; $a_{V x}-$ longitudinal acceleration of vehicle; $g$ - gravitational acceleration.

Maximum achieved vehicle deceleration during the first period of heavy braking manoeuvre is related to road surface conditions and is used as the road recognizer in the proposed control method. The variable is expressed as $\mu^{*}[16]$.

Another important state for the control method is longitudinal wheel slip $\lambda$, which is estimated from vehicle $v_{V x}$ and wheel $v_{W x}$ longitudinal velocities:

$$
\lambda=\frac{v_{W x}-v_{V x}}{v_{V x}} \cdot 100 \% \text {. }
$$

Vehicle longitudinal velocity is derived from the vehicle body deceleration signal:

$$
v_{V x}=\int a_{V x} d t \text {. }
$$

Wheel longitudinal speed is found as:

$$
v_{W x}=r_{w} \cdot \omega_{w} .
$$

\subsection{Electric vehicle model}

The EV model is completed in IPG CarMaker ${ }^{\circledR} 6.0$ (Germany) software. The 14 degrees-of-freedom model is interacted with MATLAB ${ }^{\circledR}$ from MathWorks, Inc. (USA) allowing users for rapid control algorithm development and testing. The software's integration in the HIL systems opens a great possibility for advance prototypes testing and concepts engineering, what sensitively saves development time and cost.

The sport utility EV model with all-wheel drive powertrain represents a vehicle under investigation. The specification of the vehicle parameterizations are provided by the EV's manufacturer (e.g. mass, dimensions, electric propulsion system, etc.) or are collected experimentally (e.g. suspension, tire model, etc.).

Each of four wheels is equipped with SRM. In-wheel motor transmission type is a two-stage reducer with helical gear and half-shaft. Considering SRM's peak torque (i.e. 200 $\mathrm{Nm}$ at $800 \mathrm{~V}$ ) together with overall SRM-gear ration (i.e. $1: 10.5)$, maximum torque achieved on single wheel reaches $2100 \mathrm{Nm}$. The motors behaviour is defined by the firstorder transfer function. More information about vehicle model together with its parameters is available in [16].

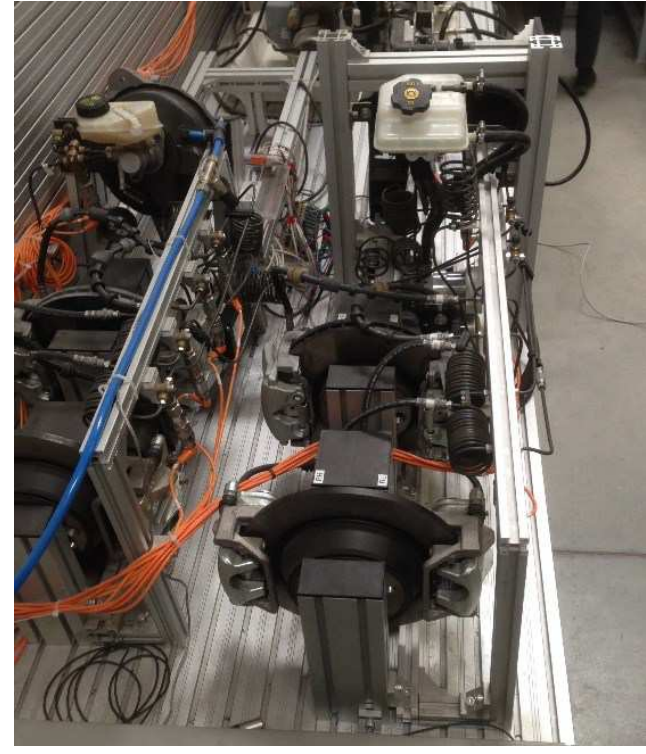

Fig. 2 Electrohydraulic brake system test bed.

The braking linings' coefficient of friction is modelled by means of a dynamic model, which was validated against data collected on the brake dynamometric test bed at Technische Universität Ilmenau (Germany). This model considers the influence of speed, pressure, and temperature on the brake linings' coefficient of friction [17].

The tire dynamics are approximated with Pacejka's "Magic Formula" with experimentally obtained coefficients. The tire-road model is a relevant element for the control method design. Particularly, it is important to know, what is the most efficient workspace for the $\lambda$ with various road surfaces? Deceleration with the optimal $\lambda$ results in maximum braking manoeuvre efficiency that impacts the deceleration distance. Moreover, when the wheel slip is equal or smaller than its corresponding peak (so-called stable region), the EV presumes steerability. On the contrary, deceleration with the $\lambda$ exceeding its optimal one (i.e. unstable zone) leads to wheels' lockage and lateral control aggravation. The ABS's task is to avoid wheel slip unstable region.

\subsection{Electrohydraulic brake system test bed}

The EHB with control unit test bed (Fig. 2), was provided by Technische Universität Ilmenau. The test rig is developed by the ZF TRW Automotive GmbH (Germany). The EHB setup is used in vehicle braking dynamics studies for reproduction of the real pressure dynamics of the brake circuit.

The vehicle model sends demanded braking pressure for each wheel to the EHB control unit. The $\mathrm{dSPACE}^{\circledR}$ (Germany) platform is utilized as an intermediate connection between the vehicle numerical model and the EHB. The requested braking pressure received from the vehicle model activates the valves that generate corresponding braking pressure between the wheels and the 
callipers. Finally, measured with the appropriate sensors line braking pressure on each wheel is returned back to the vehicle model.

\section{Blended anti-lock braking system control method}

\subsection{Control method}

The ABS control method supplies an appropriate braking torque to decelerate the vehicle with optimal wheel slip for each wheel. Different road surfaces are taken into account in control method design. A detailed description of control method and its design can be found in [16]. Only brief introduction is delivered here.

The control area network bus provides vehicle longitudinal deceleration together with wheel velocity (Fig. 3). In the next steps, applying (4) $-(7)$, two commanded variables, $\lambda$ and $\mu^{*}$, are obtained. They are used by the FLCs to generate a required torque for the actuators.

Two FLCs are designed separately for SRM and EHB control for each wheel. The fuzzy system accepts the information about vehicle body deceleration during the first step of heavy braking. Its maximum value is fed as a constant crisp input to the FLC to recognize road surface. This crisp input is uncertain, hence, computational intelligence methods, such as a fuzzy set theory, are capable to deal with such ill-defined and vague data. Thanks to the methods robustness, precise mathematical modelling may be avoided. The second input is wheel slip, which is used to decide for the requested torque $T^{r e q}$ increase or decrease.

Both FLC inputs have symmetrically dispersed over the whole universe of discourse triangular membership functions, five for $\lambda$ and seven for $\mu^{*}$. Equal sensitivity of the inputs is ensured by membership functions overlapping. The $\lambda$ is bounded in [ $\left.\begin{array}{ll}0 & 18\end{array}\right]$, and $\mu^{*}-$ in [ $\left[\begin{array}{ll}0 & 10\end{array}\right]$. Sugeno's inference method is exploited in this study. In Tab. 1, the rule base for the front and rear wheels in regenerative braking mode is provided. Considering motor's peak torque limits, the output torque for the SRM has eleven linguistic values from 0 to $200 \mathrm{Nm}$. The $T_{F B}$ is between 0 and 150 bar and has the same design principle as the RB (Tab. 1). The FLCs are designed referring to given tire model and expert's knowledge concerning efficient plant control.

Table 1 FLC rule base for front / rear wheels in regenerative mode

\begin{tabular}{ccccccc}
\hline \hline \multirow{2}{*}{$T_{R B}[\mathrm{Nm}]$} & \multicolumn{5}{c}{$\mu^{*}$} \\
\cline { 2 - 7 } & $S_{0}$ & 60 & 80 & 160 & $200 / 120$ & $200 / 140$ \\
\hline \hline & $S_{3}$ & 40 & 60 & 140 & $200 / 100$ & $200 / 120$ \\
$\lambda$ & $S_{6}$ & 20 & 40 & 120 & $200 / 80$ & $200 / 100$ \\
{$[\%]$} & $S_{9}$ & 0 & 20 & 100 & $180 / 40$ & $200 / 80$ \\
& $S_{12}$ & 0 & 0 & 60 & $160 / 20$ & $200 / 40$ \\
& $S_{15}$ & 0 & 0 & 20 & $140 / 0$ & $180 / 20$ \\
& $S_{18}$ & 0 & 0 & 0 & $120 / 0$ & $160 / 0$ \\
\hline \hline
\end{tabular}

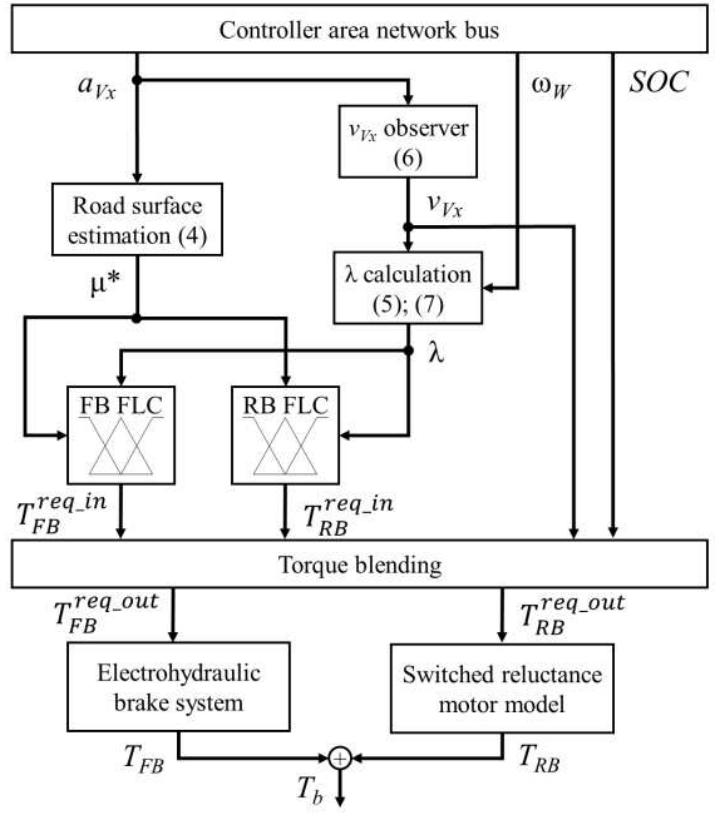

Fig. 3 Control block scheme for a single wheel of the EV: superscript ${ }^{r e q}$ stands for "requested"; RB FLC - regenerative braking fuzzy logic controller; FB FLC - friction braking fuzzy logic controller.

The modus ponens rules (If premise Then consequence) are the common expression of fuzzy input-output fit. Like in this work, the tabular representation is often acquired with trial and error method. The main criteria for Tab. 1 design is to achieve wheel slip for each tire as close as possible to the optimal one. An example of input-output linguistic mapping is as follows: If wheel "slip is $3 \%\left(S_{3}\right)$ " and road surface is "Dry", Then request from the SRM " 200 " Nm for the front wheels and " 120 " Nm for the rear wheels. The final step is to translate the output linguistic variables back to crisp numbers. For this, centre of gravity is applied.

\subsection{Torque blending}

Torque blending is realized with simple logic rules. It requires several inputs, in particular requested input $\mathrm{RB}$ and FB torques $T_{R B}{ }^{\text {req_in }}$ and $T_{F B}{ }^{\text {req_in }}{ }^{\text {in }}$, vehicle longitudinal velocity $v_{V x}$, and SOC of the battery SOC. Torque blending block outputs are requested RB torque $T_{R B}{ }^{\text {req_out }}$ for the SRM and requested FB torque $T_{F B}{ }^{\text {req_out }}$ for the EHB (Fig. 3). The approach flowchart is presented in Fig. 4. It is developed to prioritize the usage of the SRMs, yet without battery damage due to overcharge.

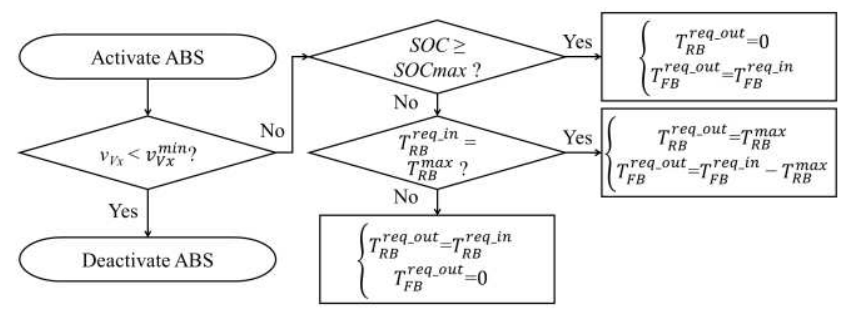

Fig. 4 Control flowchart of torque blending for a single wheel. 
Firstly, the algorithm checks the velocity of the vehicle. When vehicle longitudinal speed is slower than a desired minimum threshold $v_{V x}{ }^{\min }$ (typically $15-8 \mathrm{~km} / \mathrm{h}$ ), the ABS control is deactivated, because the distance travelled with very low speed with locked wheels is not critical.

Secondly, when the SOC reaches maximum allowed threshold $S O C_{\max }$ (e.g. $90 \%$ ), the braking switches to pure $\mathrm{FB}$ mode, where the torque for the SRM is equal to zero:

$$
\left\{\begin{array}{c}
T_{R B}^{\text {req_out }}=0 \\
T_{F B}^{\text {req_out }}=T_{F B}^{\text {req_in }} .
\end{array}\right.
$$

Thirdly, the blended ABS considers the SRM's peak performance. Specifically, when peak torque $T_{R B}{ }^{\max }$ of the SRM is requested by the FLC, the block supplies the peak torque request to the SRM and calculates additional torque for the FB actuator to ensure optimal $\lambda$ deceleration as:

$$
\left\{\begin{array}{c}
T_{R B}^{\text {req_out }}=T_{R B}^{\max } \\
T_{F B}^{\text {req_out }}=T_{F B}^{\text {req_in }}-T_{R B}^{\max }
\end{array} .\right.
$$

Finally, when none of the previous conditions are true, the EV decelerates only with SRMs as the ABS actuators:

$$
\left\{\begin{array}{c}
T_{R B}^{\text {req_out }}=T_{R B}^{\text {req_in }} \\
T_{F B}^{\text {req_out }}=0
\end{array} .\right.
$$

\section{Results}

The results of the EV heavy braking with activated ABS on a dry asphalt road $(\mu \approx 1)$ is delivered in Fig. 5. At the experiment time 3.5 seconds, the SOC of the EV's battery is assumed to exceed its upper bound. At this moment, the torque blending control is easily noticeable, because the EV switches from RB to pure FB mode. Energy recuperation is no longer conducted. The vehicle speed together with the wheels' speeds are introduced in Fig. 5. (a).

Before the controller intervention, the wheels' slip rates grow due to exceeded torque requested by the driver (i.e. the braking pedal is instantly pressed to its maximum) (Fig. 5 . (b)). Nevertheless, after the ABS activation, the wheel slip rates drop down to their optimal values for a given road surface. The optimal slips for every wheel are also depicted.

In Fig. 5. (c), the road surface estimation method is scoped. At the beginning of the braking manoeuvre, the controller measures the maximum deceleration rate of the EV body. Its peak value is mapped with an appropriate road surface. The blue line symbolizes the crisp input for the estimation road conditions. The variable together with wheel slip is therefore processed by the FLC to estimate a relevant braking torques.

In Fig. 5. (d), braking torques for each in-wheel SRM of the EV are presented. Until the SOC makes an impact on blended ABS, it is seen that the SRM supplies its maximum available torque for the front wheels. As a result, the torque blending requests additional torque from the EHB (Fig. 5. (e)) to lead $\lambda$ as close as possible to their theoretical optimal values in accordance to (9). For the rear wheels, however, the generated torques by the SRMs (Fig. 5. (d)) are enough to reach optimal rate. Thus, the FB torques are not required (Fig. 5. (e)) as stated by (10).

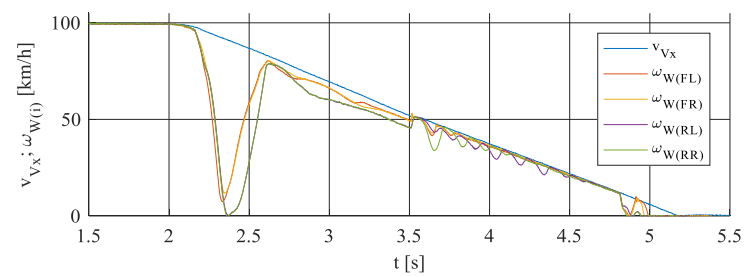

(a)

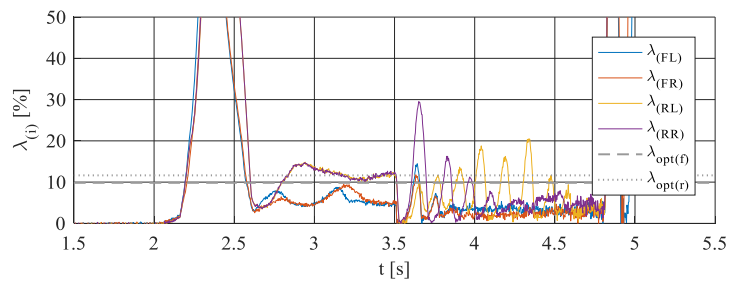

(b)

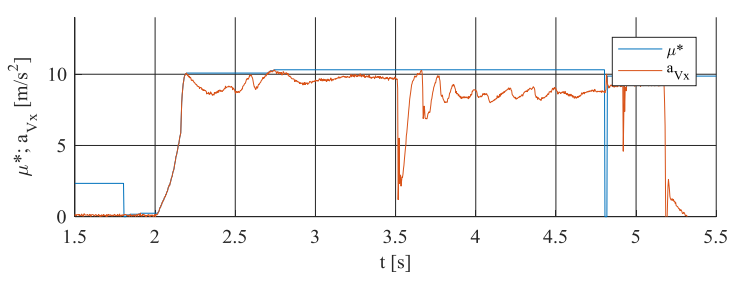

(c)

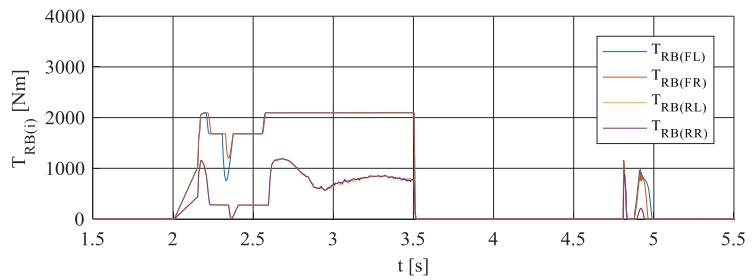

(d)

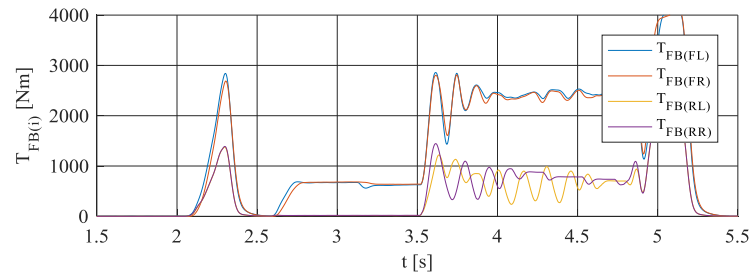

(e)

Fig. 5 Experimental results from vehicle braking on a high- $\mu(\mu \approx 1)$ road surface: (a) vehicle and wheels speeds; (b) wheels longitudinal slips; (c) road recognition with vehicle body deceleration rate (d) FB torques; (e) RB torques; [FL - front left, FR - front right, RL - rear left, $\mathrm{RR}$ - rear right]

When the SOC overshoot steps in (i.e. $t=3.5 \mathrm{~s}$ ), the energy regeneration stops, and the SRMs are not used as braking 
actuators any more (Fig. 5. (d)). Consequently, the RB torques for all wheels drop to zero. On the contrary, the system moves to the pure FB mode. Now, only the EHB's torques are applied to decelerate the transport (Fig. 5. (e)), applying (8).

Moreover, the FB torques are not as smooth as RB ones. Furthermore, the optimal wheel slip achievability is not as precise as in the case, when the SRMs affect vehicle deceleration (Fig. 5. (b)). This phenomenon is mainly due to the EHB significant delay as well as the plant complexity (i.e. wheel tire highly nonlinear behaviour). It was also studied by the authors in the previous work [16].

The conclusion was made that thanks to the electric drives' fast response, the control method accomplishes fast and more accurate control. As a result, the EV's RB braking affords noticeably more efficient EV deceleration performance under the ABS operation. It allows for vehicle stopping distance diminishment. However, the electric motors are not always fully available as the braking systems, as for instance in the case of deceleration on high- $\mu$ surfaces or in case with the exceeded SOC threshold.

\section{Conclusions}

In this paper, the blended ABS control method for all-wheel drive sport utility EV is described. The SOC and the requested torques from both actuators, namely in-wheel SRMs and EHB, are taken into consideration for providing a sufficient braking torque to presume maximum deceleration efficiency for every wheel independently. The efficiency is guaranteed by the optimal wheel slip ratio braking for each separate wheel. In combination with the intelligent FLC, the blended ABS control method provides high efficiency and robustness against varying road conditions and changing system states.

The proposed solution is verified against HIL simulation, where the experimentally validated EV is coupled with EHB test bed, which provides real brakes dynamics followed by significant hardware delay. The presented experimental results are dedicated to heavy braking conditions on a high$\mu$ road surface, during which the upper SOC threshold is achieved. As a result, the blended ABS switches from the $\mathrm{RB}$ mode to the pure FB, and the vehicle continues the deceleration process with optimal slip ratio without motors' impact and performance degradation.

Despite good optimal slip control for various road surfaces, the proposed FLC-based control method has several limitations. Firstly, the method depends on tire model, whose behaviour is also different for other types of vehicle characterized by different centres of gravity, masses, etc. Hence, for other vehicle and tire types the control method must be slightly modified. Secondly, to design an FLC for complex control system, like ABS, expert's multidisciplinary knowledge is essential.
Acknowledgements This project has received funding from the European Union's Horizon 2020 research and innovation program under grant agreement No. 675999.

\section{References}

1. M. Ehsani, Y. Gao, S. E. Gay, and A. Emadi, Modern Electric, Hybrid Electric, and Fuel Cell Vehicles, Boca Raton, Florida, USA: CRC Press, 2005.

2. A. Emadi, Handbook of Automotive Power Electronics and Motor Drives, Boca Raton, Florida, USA: Taylor and Francis Group, 2005.

3. V. Ivanov, "A review of fuzzy methods in automotive engineering applications,” Eur. Transp. Res. Rev., Vol. 7, pp. 110, 2015.

4. X. Zhang, Y. Wang, G. Liu, and X. Yuan, "Robust regenerative charging control based on T-S fuzzy sliding-mode approach for advanced electric vehicle," IEEE Trans. on Transp. Electrif., Vol. 2, pp. 52-65, 2016.

5. G. Xu, W. Li, K. Xu, and Z. Song, "An intelligent regenerative braking strategy for electric vehicles," Energies, Vol. 4, pp. 14611477, 2011.

6. J. Zhang, B. Song, S. Cui, J. Zhang, and D. Ren, "Fuzzy logic approach to regenerative braking system," 2009 Intern. Conf. on Intel. Human-Machine Syst. And Cybern., pp. 451-454, 2009.

7. X. Li, L. Xu, L. Hua, J. Li, and M. Ouyang, "Regenerative braking control strategy for fuel cell hybrid vehicles using fuzzy logic," 2008 Intern. Conf. on Elect. Machines and Syst., pp. 2712-2716, 2008.

8. X. Nian, F. Peng, and H. Zhang, "Regenerative braking system of electric vehicle driven by brushless DC motor," IEEE Trans. on Indust. Elect., Vol. 61, pp. 5798-5808, 2014.

9. D.-H. Kim, J.-M. Kim, S.-H. Hwang, and H.-S. Kim, „Optimal brake torque distribution for a four-wheel-drive hybrid electric vehicle stability enhancement,“ Proc. IMehE Part D: J. Automobile Engineering, Vol. 221, pp. 1357-1366, 2007.

10. D. Paul, E. Velenis, D. Cao, and T. Dobo, "Otpimal $\mu$-estimation based regenerative braking strategy for an AWD HEV," IEEE Trans. on Transp. Electrif., Vol. 3, pp. 249-258, 2017.

11. D. Savitski, V. Ivanov, B. Shyrokau, T. Pütz, J. de Smet, and J. Theunissen, "Experimental investigation on continuous regenerative anti-lock braking system of full electric vehicle," Int. J. Automotive Technology, Vol. 17, pp. 327-338, 2016.

12. P. Dong, Z. Jianwu, and Y. Chengliang, "Regenerative braking control system improvement for parallel hybrid electric vehicle," Intern. Tech. and Innov. Conf. 2016 (ITIC 2006), pp. 1902-1908, 2006.

13. J. Guo, X. Jian, and G. Lin,, "Performance evaluation of an antilock braking system for electric vehicles with a fuzzy sliding mode controller," Energies, vol. 7, pp. 6459-6476, 2014.

14. B. Shyrokau, D. Wang, D. Savitsky, and V. Ivanov, "Vehicle dynamics control with energy recuperation based on control allocation for independent wheel motors and brake system," Int. J. Powertrain, Vol. 2, pp. 153-181, 2013.

15. A. Aksjonov, V. Vodovozov, and Z. Raud, "Improving energy recovery in blended antilock braking systems of electric vehicles," 16th Inter. Conf. of Indust. Informat. (INDIN'2018), pp. 589-594, 2018.

16. A. Aksjonov, V. Vodovozov, K. Augsburg, and E. Petlenkov, "Design of regenerative anti-lock braking system controller for 4-in-wheel-motor drive electric vehicle with road surface estimation," Int. J. Automotive Technology, Vol. 19, pp. 727$742,2018$.

17. V. Ricciardi, D. Savitski, K. Augsburg, and V. Ivanov, "Estimation of brake friction coefficient for blending function of base braking control," SAE Int. J. Passeng. Cars - Mech. Syst., Vol. 10, pp. 774-785, 2017. 